\title{
LINDELÖF PROPERTY IN FUNCTION SPACES AND A RELATED SELECTION THEOREM
}

\author{
WITOLD MARCISZEWSKI \\ (Communicated by Dennis Burke)
}

\begin{abstract}
Let $X$ be a separable metrizable space. If $K$ is a compact space whose function space $C(K)$ is weakly $K$-analytic, then the space $C_{p}(X, K)$ of continuous maps from $X$ to $K$ with the pointwise topology has the Lindelöf property. If $E$ is a Banach space whose weak topology is $\mathcal{K}$-analytic, then each lower semicontinuous map from $X$ to the family of nonempty closed convex subsets of the unit ball of the dual $E^{*}$ with the weak*-topology admits a continuous selection. This extends some results of Corson and Lindenstrauss.
\end{abstract}

1. Introduction. In this paper we extend some results of Corson and Lindenstrauss $[2,3]$ concerning weakly compact sets in Banach spaces to the case of compact spaces $K$ whose function spaces $C(K)$ are $\mathcal{K}$-analytic in the weak topology (for these notions and some related basic facts we refer the reader to $\S 2$ ). In particular, if $K$ is such a compact space and $X$ is a separable metrizable space, then the space $C_{p}(X, K)$ of continuous functions from $X$ to $K$ with the pointwise topology has the Lindelöf property (Theorem 4.1). This allows one to obtain the following selection theorem (Theorem 5.1): If $E$ is a weakly $\mathcal{K}$-analytic Banach space, then each lower semicontinuous map from a separable metrizable space to the family of nonempty closed convex subsets of the unit ball of the dual $E^{*}$ equipped with the weak*-topology admits a continuous selection.

We also construct a compact subspace $K$ of the $\Sigma$-product of the real line such that $C_{p}(X, K)$ is not Lindelöf, and $E=C(K)$ does not satisfy the assertion of the second proposition, $X$ being the unit interval $(\S 6)$.

The proof of our results is based on a recent characterization of compact spaces $K$ whose function spaces are $K$-analytic, due to Mercourakis [6] (cf. also Sokolov [10] (see §3)), and on the original results of Corson and Lindentrauss.

I would like to thank R. Pol for valuable discussions on the subject of this paper.

2. Notation and terminology. Our terminology follows Engelking [4] and Negrepontis [9]. We denote by $R$ the real line and by $\omega^{\omega}$ the space of irrationals equipped with a standard complete metric $d$ (cf. [4, Example 4.2.12]). Given topological spaces $X$ and $Y, C_{p}(X, Y)$ is the space of all continuous functions from $X$ to $Y$ with the topology of pointwise convergence; we shall write $C_{p}(X)$ instead of $C_{p}(X, R)$. All topological spaces considered in this paper are assumed to be completely regular.

2.1. Eberlein compacta and Corson compacta. Given a set $\Gamma$, the $\Sigma$-product of the real line is the subspace $\Sigma(\Gamma)$ of the Tychonoff product $R^{\Gamma}$ consisting of points

Received by the editors June 2, 1986.

1980 Mathematics Subject Classification (1985 Revision). Primary 54C35, 54C65; Secondary $46 \mathrm{E} 10$. 
with all but countable many coordinates equal to 0 [4, Problem 2.7.13], and let $c_{0}(\Gamma)=\left\{x \in R^{\Gamma}:\right.$ for each $\varepsilon>0$ the set $\{\gamma:|x(\gamma)|>\varepsilon\}$ is finite $\} \subset \Sigma(\Gamma)$.

Eberlein (Corson) compacta are compact spaces which can be embedded in some $c_{0}(\Gamma)(\Sigma(\Gamma))$; the class of Eberlein compacta coincides with the class of weakly compact subsets of Banach spaces (cf. [9]).

2.2. K-analytic spaces. A topological space $X$ is $K$-analytic if there exists an upper semicontinuous map $\varphi$ from $\omega^{\omega}$ to the family of compact subsets of $X$ such that

$$
\varphi\left(\omega^{\omega}\right)=\bigcup\left\{\varphi(\sigma): \sigma \in \omega^{\omega}\right\}=X
$$

(cf. [5]).

For each Eberlein compactum $K, C(K)$ is $\mathcal{K}$-analytic in the weak topology and each compact space $K$ with weakly $K$-analytic function space $C(K)$ (i.e. in the terminology of [9], a Talagrand compact space) is a Corson compactum (see [9, Theorems 6.9, 6.10,6.23]).

2.3. The space $c_{o}\left(\Gamma, C_{p}(X)\right)$. Let $\Gamma$ be a set. We say that a function $f$ from $\Gamma$ to $C_{p}(X)$ vanishes at infinity if for each neighborhood $U$ of 0 in $C_{p}(X), f(\gamma)$ belongs to $U$ for all but finitely many $\gamma \in \Gamma$. By $c_{0}\left(\Gamma, C_{p}(X)\right)$ we denote the space of all functions from $\Gamma$ to $C_{p}(X)$ which vanish at infinity, endowed with the topology of pointwise convergence.

2.4. The sets in $E^{\Gamma}$ invariant under projections. Let $E$ be a linear space and let $\Delta$ be a subset of a set $\Gamma$. A map $\Pi_{\Delta}: E^{\Gamma} \rightarrow E^{\Gamma}$ assigns to each function $f$ from $\Gamma$ to $E$ a function $\Pi_{\Delta}(f)$ which agrees with $f$ on $\Delta$ and takes the value 0 for $\gamma \in \Gamma \backslash \Delta$. A subset $A$ of $E^{\Gamma}$ is said to be invariant under projection if $\Pi_{\Delta}(A) \subset A$ for all $\Delta \subset \Gamma$ (cf. [2]).

3. A characterization by Mercourakis of compacta with $K$-analytic function spaces. Given a topological space $X$ we define, following Mercourakis [6],

$$
\begin{aligned}
C_{1}(X)=\{f: X \rightarrow R: & f \text { is bounded and for every } \varepsilon>0 \text { the set } \\
& \{x \in X:|f(x)|>\varepsilon\} \text { is closed and discrete in } X\} .
\end{aligned}
$$

Observe that if $S$ is a separable metrizable space and $M$ is a compact space, then $C_{1}(S \times M) \subset \Sigma(S \times M)$. We consider $C_{1}(X)$ with the pointwise topology.

3.1. THEOREM (MERCOURAKIS [6, THEOREM 3.2]). For a compact space $K, C(K)$ is weakly $K$-analytic if and only if $K$ embeds in $C_{1}\left(\omega^{\omega} \times M\right)$ for some compact space $M$.

3.2. THEOREM (MERCOURAKIS [6, THEOREM 4.2]). Let E be a Banach space whose weak topology is $\mathcal{K}$-analytic. Then there exist a compact space $M$ and a linear continuous injection $T:\left(E^{*}, w^{*}\right) \rightarrow C_{1}\left(\omega^{\omega} \times M\right), w^{*}$ being the weak $k^{*}$ topology in the dual space $E^{*}$.

4. Lindelöf property for the spaces of continuous functions. The following result is a generalization of a theorem of Corson and Lindenstrauss [2, Theorem $2.3]$.

4.1. THEOREM. Let $X$ be a topological space which is the continuous image of a separable metrizable space and let $K$ be a compact space whose function space 
$C(K)$ is weakly $K$-analytic. Then the function space $C_{p}(X, K)$ has the Lindelof property.

ProOF. By Mercourakis' theorem (see $\S 3$ ), we can assume that $K$ is a subset of the space $C_{1}\left(\omega^{\omega} \times M\right)$ for some compact space $M$. Let $p: Y \rightarrow X$ be a continuous map from a separable metrizable space $Y$ onto the space $X$, and let $\hat{Y}$ be a completion of the space $Y$. Let us consider a function $f \in C_{p}(X, K)$ and let $S=\left\{(\sigma, m) \in \omega^{\omega} \times M:(\exists x \in X) f(x)(\sigma, m) \neq 0\right\}$. Since $C_{1}\left(\omega^{\omega} \times M\right)$ is contained in the $\Sigma$-product $\Sigma\left(\omega^{\omega} \times M\right)$, the set $S$ is at most countable, the space $X$ being separable. Let $\left\{\left(\sigma_{i}, m_{i}\right): 1 \leq i \leq \alpha\right\}$ be an enumeration of $S$ for some $\alpha \leq \omega$. Let $\hat{f}: \omega^{\omega} \times M \rightarrow C_{p}\left(\omega^{\omega} \times Y\right)$ be defined by the formula

$$
\hat{f}(\sigma, m)(\tau, y)=\left\{\begin{array}{l}
f(p(y))\left(\sigma_{i}, m\right) \cdot \max \left(i\left(i^{-1}-d\left(\tau, \sigma_{i}\right)\right), 0\right), \quad \text { if }(\sigma, m)=\left(\sigma_{i}, m_{i}\right), \\
0 \quad \text { if }(\sigma, m) \notin S,
\end{array}\right.
$$

for $\sigma, \tau \in \omega^{\omega}, m \in M, y \in Y$.

We check that

$$
\hat{f} \in c_{0}\left(\omega^{\omega} \times M, C_{p}\left(\omega^{\omega} \times Y\right)\right) .
$$

Fix $(\tau, y) \in \omega^{\omega} \times Y$ and $\varepsilon>0$ and let $T=\{(\sigma, m):|\hat{f}(\sigma, m)(\tau, y)|>\varepsilon\} \subset S$. If $\left(\sigma_{i}, m_{i}\right) \in T$ then $\left|f(p(y))\left(\sigma_{i}, m_{i}\right)\right|>\varepsilon$, hence the set $T$ is closed and discrete in $\omega^{\omega} \times M$. Since $d\left(\tau, \sigma_{i}\right)<i^{-1}$ for $\left(\sigma_{i}, m_{i}\right) \in T$, the set $T$ is contained in the compact set $\left(\{\tau\} \cup\left\{\sigma_{i}: d\left(\tau, \sigma_{i}\right)<i^{-1}\right\}\right) \times M$. Therefore the set $T$ is finite. This proves (2).

Let us consider the smallest subspace $A$ of $c_{0}\left(\omega^{\omega} \times M, C_{p}\left(\omega^{\omega} \times Y\right)\right)$ invariant under projections (see $\S 2.4)$ containing the set of all functions $\hat{f}$ which we have associated with functions $f \in C_{p}(X, K)$; i.e.

$$
A=\left\{\Pi_{W} \circ \hat{f}: f \in C_{p}(X, K), W \subset \omega^{\omega} \times M\right\} .
$$

The space $A$ satisfies the assumptions of Lemma 2.1 from Corson and Lindenstrauss [2]. To verify this, we have only to make sure that given a function $f \in$ $C_{p}(X, K)$, there exists a $G_{\delta}$-set $Z$ in $\hat{Y}$ and a function $\hat{h} \in c_{0}\left(\omega^{\omega} \times M, C_{p}\left(\omega^{\omega} \times Z\right)\right)$ such that $Y \subset Z$ and $\hat{h}(\sigma, m)(\tau, y)=\hat{f}(\sigma, m)(\tau, y)$ for every $\sigma, \tau \in \omega^{\omega}, m \in M$, and $y \in Y$.

Let $g=f \circ p: Y \rightarrow K$. The range of $g$ is contained in the compact metrizable space $\Pi_{S}(K)$ and therefore, by Lavrentieff's theorem [4, Theorem 4.3.20], $g$ can be extended to a continuous function $h: Z \rightarrow \Pi_{S}(K)$ defined on a $G_{\delta}$-set $Z$ in $\hat{Y}$ containing $Y$. The function $\hat{h}$ defined by formula (1) (with $f$ replaced by $h$ and $y \in Z$ ) has the desired properties.

Therefore, by the lemma of Corson and Lindenstrauss we infer that $A$ is a Lindelöf space.

We shall complete the proof, defining a continuous map

$$
\varphi: A \rightarrow C_{p}\left(X, C_{1}\left(\omega^{\omega} \times M\right)\right)
$$

whose range contains the space $C_{p}(X, K)$ as a closed subspace. To this end let $q: X \rightarrow Y$ select for each $x \in X^{-}$a point $q(x)$ from $p^{-1}(x)$, and let

$$
\varphi(g)(x)(\sigma, m)=g(\sigma, m)(\sigma, q(x)) \quad \text { for } g \in A, x \in X, \sigma \in \omega^{\omega}, m \in M .
$$


The map $\varphi$ is continuous and, since $\varphi\left(\Pi_{W} \circ \hat{f}\right)=\Pi_{W} \circ f$ by (1), the range of $\varphi$ contains the space $C_{p}(X, K)$. The observation that, by compactness of $K, C_{p}(X, K)$ is a closed subset of $C_{p}\left(X, C_{1}\left(\omega^{\omega} \times M\right)\right)$ completes the proof of the theorem.

4.2. REMARK. Let $X$ be a continuous image of a complete separable metrizable space and let $M$ be a compact space. Using the method from the proof of Theorem 4.1 one can show that if $A$ is a subset invariant under projections of $C_{1}\left(\omega^{\omega} \times M\right)$, then the space $C_{p}(X, A)$ is Lindelöf.

5. A selection theorem. In this section we shall follow the reasoning of Corson and Lindenstrauss [3, proof of Theorem 2.2] to derive from Theorem 4.1 the following result on continuous selections, extending [3, Theorem 2.2]. (We refer the reader to [ 7 or 8 ] for some standard terminology related to the selection theory, in particular we say that the map $\varphi$ from a set $A$ to the family of subsets of a Banach space $E$ is bounded if the set $\varphi(A)=\bigcup\{\varphi(a): a \in A\}$ is bounded in $E$.)

5.1. THEOREM. Let $X$ be a topological space which is a continuous image of a separable metrizable space and let $E$ be a Banach space whose weak topology is $K$-analytic. Then each lower semicontinuous locally bounded map $\varphi$ from $X$ to the family of all nonempty closed convex subsets of the dual space $E^{*}$ endowed with the weak $k^{*}$-topology admits a continuous selection, i.e. there is a continuous map $f: X \rightarrow\left(E^{*}, w^{*}\right)$ with $f(x) \in \varphi(x)$ for all $x \in X$.

The proof of Theorem 5.1 is based on the following lemma.

5.2. LEMMA. Let $X$ be as in Theorem 5.1 and let $\varphi$ be a lower semicontinuous map from $X$ to the family of nonempty closed convex subsets of a compact subspace $K$ of the space $C_{1}\left(\omega^{\omega} \times M\right)$, where $M$ is compact ( $\left.c f . \S 3\right)$. Then $\varphi$ has a continuous selection.

Proof (CF. [3, ThEOREM 2.2]). Let $L=\left\{f \in C_{1}\left(\omega^{\omega} \times M\right):|f| \leq|g|\right.$ for some $g \in K\}$ (where $|f| \leq|g|$ means that $|f(\sigma, m)| \leq|g(\sigma, m)|$ for each $(\sigma, m) \in$ $\left.\omega^{\omega} \times M\right)$. One easily checks that $L$ is compact (cf. [10, Lemma 3]).

Let $S$ be the family of all finite subsets of $\omega^{\omega} \times M$. For every $s \in S$, the set $F_{s}=\left\{f \in C_{p}(X, L): \Pi_{s}(f(x)) \in \Pi_{s}(\varphi(x))\right.$ for all $\left.x \in X\right\}$ is closed in the space $C_{p}(X, L)$. We show that the family $\left\{F_{s}: s \in S\right\}$ has nonempty intersection. Since by Theorem 4.1 the space $C_{p}(X, L)$ is Lindelöf it is enough to prove that for every countable subset $\tau$ of $S$ the family $\left\{F_{s}: s \in T\right\}$ has nonempty intersection. Let $T=\bigcup\{s: s \in \tau\}$.

We can consider $\Pi_{T}(L) \subset L$ as a subset of a complete metrizable locally convex space $R^{T}$. Since the map $\varphi_{T}=\Pi_{T} \circ \varphi: X \rightarrow R^{T}$ is lower semicontinuous and its values are nonempty compact convex sets, by Michael's Selection Theorem [7, Theorem 3.2] there is a continuous selection $f$ for $\varphi_{T}(X$ is paracompact, being a continuous image of a Lindelöf space). Evidently $f \in \bigcap\left\{F_{s}: s \in T\right\}$.

Now, it can be easily checked that each member of $\bigcap\left\{F_{s}: s \in S\right\}$ is a continuous selection for $\varphi$.

ProOF OF THEOREM 5.1. Let $T:\left(E^{*}, w^{*}\right) \rightarrow C_{1}\left(\omega^{\omega} \times M\right)$ be a continuous injection given by Theorem 3.2 of Mercourakis.

Let $\left\{U_{\alpha}: \alpha<\beta\right\}$ be a locally finite open cover of $X$, such that for each $\alpha<\beta$ the set $\bigcup\left\{\varphi(x): x \in U_{\alpha}\right\}$ is bounded in $E^{*}$ and hence its weak ${ }^{*}$ closure is compact. 
Applying Lemma 5.2 to the map $\left.T \circ \varphi\right|_{U_{\alpha}}$ where $\left.\varphi\right|_{U_{\alpha}}$ is the restriction of $\varphi$ to $U_{\alpha}$, we get a continuous selection $f_{\alpha}$ for $\left.T \circ \varphi\right|_{U_{\alpha}}$ and, the restriction of $T$ to any bounded set in $E^{*}$ being a homeomorphic embedding, the map $T^{-1} \circ f_{\alpha}$ is a continuous selection for $\left.\varphi\right|_{U_{\alpha}}$. Now, the selection we are looking for can be defined by the formula $f=\sum_{\alpha<\beta} p_{\alpha} \cdot T^{-1} \circ f_{\alpha}$, where $\left\{p_{\alpha}: \alpha<\beta\right\}$ is a partition of unity subordinated to $\left\{U_{\alpha}: \alpha<\beta\right\}$.

6. An example. The example discussed in this section shows that possible generalizations of the results obtained in this paper cannot go too far. The idea of our construction is similar to that given by Mercourakis [6, Theorem 5.8].

6.1. EXAMPLE. There is a compact convex subspace $L$ of the $\Sigma$-product $\Sigma(I)$, $I$ being the unit interval (see $\S 2.1$ ), such that

(a) the function space $C_{p}(I, L)$ is not Lindelöf,

(b) there is a lower semicontinuous map $\varphi$ from $I$ to the family of nonempty closed convex subsets of $L$ which does not admit any continuous selection; in particular the assertion of Theorem 5.1 fails for the Banach space $E=C(L)$.

Let $S$ be the collection of $n$-tuples $\left\langle t_{1}, t_{2}, \ldots, t_{n}\right\rangle \in I^{n}$ such that $\left|t_{i}-t_{i+1}\right| \leq 2^{-i}$ for $i=1,2, \ldots, n, n=1,2, \ldots$, and let $A$ be the collection of all subsets $A$ of $I$ such that each finite $S \subset A$ is contained in $\left\{t_{1}, t_{1}, \ldots, t_{n}\right\}$ for some $\left\langle t_{1}, t_{2}, \ldots, t_{n}\right\rangle \in S$. Let $\chi_{A}$ be the characteristic function of the set $A \subset I$ and let $K=\left\{\chi_{A}: A \in A\right\}$ be considered as a subspace of the Tychonoff cube $\{0,1\}^{I}$. The definition of $K$ being of finite character, $K$ is a compact space.

The space $L$ we are looking for is the closed convex hull of $K$ in $I^{I}$.

Firstly, we check that $L \subset \Sigma(I)$. If $A \in A$, then $A$ has at most one limit point, so $A$ is countable. Hence all functions $\chi_{A}$ in $K$ are of the first Baire class, and $L$ consists of functions of the first Baire class as well by [1, Theorem $5 \mathrm{E}]$ and therefore each $f \in L$ is in the closure of a countable subset of the convex hull of $K$ $[\mathbf{1}$, Theorem $3 \mathrm{~F}]$, i.e. $f \in \Sigma(I)$.

We shall now define a map $\varphi$ satisfying (b). For each $t \in I$, let $S_{t}$ be the collection of $n$-tuples $\left\langle t_{1}, t_{2}, \ldots, t_{n}\right\rangle \in S$ such that $t_{n}=t$. Let $A_{t}=\{A \in A: t \in A$ and all finite subsets of $A$ are contained in some set $\left\{t_{1}, t_{2}, \ldots, t_{n}\right\}$ with $\left.\left\langle t_{1}, t_{2}, \ldots, t_{n}\right\rangle \in S_{t}\right\}$ and let $\psi(t)=\left\{\chi_{A}: A \in A_{t}\right\}$; notice that $\psi(t)$ is a compact set. We shall show that the map $t \rightarrow \psi(t)$ is lower semicontinuous.

Let $U$ be an open set in $\{0,1\}^{I}$, let $\chi_{A} \in \psi(t) \cap U$ for some $t \in I$, and let us choose finite sets $S \subset A$ and $T \subset I \backslash A$ such that the basic neighborhood $W=\left\{f \in\{0,1\}^{I}: S \subset f^{-1}(1) \subset I \backslash T\right\}$ is contained in $U$. There exists an $n$-tuple $\left\langle t_{1}, t_{2}, \ldots, t_{n}\right\rangle \in S_{t}$ (in particular $t_{n}=t$ ) with $S \subset\left\{t_{1}, t_{2}, \ldots, t_{n}\right\}$. Let $V=\left(t-2^{-n}, t+2^{-n}\right) \backslash T$. If $s \in V$, then $\left\langle t_{1}, t_{2}, \ldots, t_{n}, s\right\rangle \in S_{s}$, so for $B=\left\{t_{1}, t_{2}, \ldots, t_{n}, s\right\} \backslash T$ we get $\chi_{B} \in \psi(s) \cap W$.

Let $\varphi(t)$ be the closed convex hull of $\psi(t)$. Then $t \rightarrow \varphi(t)$ is lower semicontinuous (cf. [7, Propositions 2.3 and 2.6]). Given a continuous map $h: I \rightarrow L$ there is a countable set $T \subset I$ such that all functions $h(s)$ for $s \in I$ vanish outside of $T$, but on the other hand, if $h(s) \in \varphi(s)$, then $h(s)(s)=1$ and therefore there is no continuous selection for $\varphi$.

Property (a) of $L$ follows from (b). If $C_{p}(I, L)$ were a Lindelöf space, then the reasoning from the proof of Lemma 5.2 would give a continuous selection for $\varphi$. 


\section{REFERENCES}

1. J. Bourgain, D. H. Fremlin, and M. Talagrand, Pointwise compact sets of Baire-measurable functions, Amer. J. Math. 100 (1978), 845-886.

2. H. H. Corson and J. Lindenstrauss, On function spaces which are Lindelöf spaces, Trans. Amer. Math. Soc. 121 (1966), 476-491.

3. $\_$, Continuous selections with nonmetrizable range, Trans. Amer. Math. Soc. 121 (1966), 492-504.

4. R. Engelking, General topology, PWN, Warszawa, 1977.

5. J. E. Jayne and C. A. Rogers, K-analytic sets, Analytic Sets, Academic Press, New York, 1980.

6. S. Mercourakis, On weakly countably determined Banach spaces, Trans. Amer. Math. Soc. 300 (1987), 307-327.

7. E. Michael, Continuous selections. I, Ann. of Math. (2) 63 (1956), 361-382.

8. J. Nagata, Modern general topology, North-Holland, Amsterdam, 1968.

9. S. Negrepontis, Banach spaces and topology, Handbook of Set-Theoretic Topology, NorthHolland, Amsterdam, 1984, pp. 1045-1142.

10. G. A. Sokolov, On some classes of compact spaces lying in $\Sigma$-products, Comm. Math. Univ. Carolinae 25 (1984), 219-231.

Department of Mathematics, University of WarsaW, PkiN IX P., 00-901, WaRSZAWA, POLAND 